\title{
EVALUATION OF VASOMOTOR RESPONSE IN OPHTHALMIC MIGRAINE PATIENTS
}

\author{
Adriana Bulboaca, Corina Ursu, A. Bulboaca \\ Neurol ogy Department, Rehabilitation Hospital, University of Medicineand Pharmacy, \\ Cluj-Napoca, Romania
}

\begin{abstract}
Introduction. Hemodynamic changes of the cerebral arteries in ophthalmic migraine may be an important pathophysiological mechanism of the disease. The nitric oxide (NO) synthesis plays a significant role in hemodynamic changes due to vasodilatation effect. The purpose of this study is to evaluate the possible hemodynamic changes by Doppler measurements and their correlation with NO synthesis in ophthalmic migraine.

Method. 37 ophthalmic migraine patients were compared with 35 healthy volunteers. Other cerebral and ophthalmic diseases were excluded by ophthalmological, neurological, and cranial MRI examinations. The migraine assessment scale (MIDAS) was used for clinical evaluation. Transcranial Doppler ultrasonography (TCD) was performed using 2 and $4 \mathrm{MHz}$ frequencies transducers. The flow velocities of the internal carotid artery, middle cerebral artery, posterior cerebral artery, and NO plasma concentration were assessed 2 hours after migraine attacks.

Results. The NO synthesis was significantly increased compared to the control group. The flow velocities decreased in internal carotid artery, middle cerebral artery, and posterior cerebral artery. The correlation between decreased flow velocities and NO plasma concentration was significant.

Conclusions. Autonomic nervous system dysfunction in ophthalmic migraine is an important pathophysiological mechanism. The flow velocity status may reflect the vasomotor response and endothelial reactivity induced by NO synthesis. Further studies on the vasomotor response are necessary for the evaluation of this pathophysiological mechanism implication in ophthalmic migraine.
\end{abstract}

Keywords: migraine, nitric oxide, transcranial Doppler ultrasonography

\section{INTRODUCTION}

Hemodynamic changes of the cerebral arteries in ophthalmic migraine may be an important pathophysiological mechanism of the disease. Depending on the signal perceived by endothelial cells, it can result in a vasoconstrictor or vasodilator effect. The nitric oxide (NO) synthesis plays a significant role in hemodynamic changes due to vasodilatation effect (1). NO is well known for its ability to relax blood vessels in addition to other biological effects. One of the most important harmful effects is its contribution to nitro-oxidative stress (2). Therefore, the increased synthesis of NO may lead to cells damage due to nitro-oxidative stress, despite of its vasodilatation benefic effect that contributes to adequate blood flow into the tissues. The synthesis of NO from L-arginine is controlled by nitricoxide synthase (NOS) which requires NADPH and molecular oxygen (3). Constitutional forms of NOS are endothelial nitric oxide synthase (eNOS) and neuronal nitric oxide synthase (nNOS) (4). In pathological conditions such is inflammation, an inducible form of NOS (iNOS) is produced during the inflammatory process being able to modulate biosynthesis of prostaglandins through non-COX-related pathways (5). In these instances the increasing synthesis of NO can exceed the physiological quantities that serve for vasodilatation and adequate blood flow, contributing to the onset of nitro-oxidative stress and stimulating the pain transmission (6). NO may cause activity alterations on different levels of the trigeminal system including enhancement of calcitonin gene related protein (CGRP) release. Both mediators (NO and CGRP) facilitate nociceptive transmission, possibly via presynaptic mechanisms (7). NO itself is a short-lived, highly 
reactive species, but its major products nitrate and nitrite (collectively termed NOx) can readily measured retrospectively in biological samples as markers of NO production (8). The decreasing production of NO is a biomarker for endothelial dysfunction, and increasing productions is associated with nitro-oxidative stress $(9,10)$.

Trascranial Doppler ultrasonography (TDU) represents a noninvasive modern method of determining the flow velocities in cerebral arteries. The flow velocities in cerebral arteries can be a good indicator for cerebral circulation in various pathological circumstances associated with occlusive or vasospastic mechanisms or with pathological vasodilatation mechanism (11). This method is of particular value for the detection of pathological variation of blood flow due to permanent lesions as are atherosclerosis plaques or anevrisma, or due to episodic variation of vessel wall diameter due to vasospasm (12). The functional parameters that characterize the blood circulation in cerebral arteries can be correlated with biological marker in order to a better characterization of pathophysiological mechanism associated with various vascular cerebral pathologies. Vascular changes associated with migraine are considered to involve selectively cranial blood vessels and is found to share the same risk factors as cardiovascular disease (13). The mechanisms underlying the relation between migraine and cardiovascular risk factors remain unclear but include the impairment of large vessels compliance (14).

The purpose of this study is to evaluate the possible hemodynamic changes by Doppler measurements and their correlation with NO synthesis in ophthalmic migraine.

\section{METHOD}

\section{Study groups and protocol}

The study had the local Ethics Committee approval (Clinical Rehabilitation Hospital, Cluj-Napoca, Romania) and the Informed Consent for each patient. 37 ophthalmic migraine patients were compared with 35 healthy volunteers. Ophthalmic migraine (or retinal migraine) was suspected when the patient suffered of repeated episodes of short-lasting, diminished vision or blindness that preceded or accompanied a headache (15). The attacks of ophthalmic migraine (unlike a migraine aura that affect the vision) affected only one eye, not both (16). Pathological other ophthalmological conditions were excluded by ophthalmologic examination.
Other cerebral diseases were excluded by neurologic, carotid Doppler ultrasound, and cranial MRI examinations. The migraine assessment scale (MIDAS) was used for clinical evaluation. Transcranial Doppler ultrasonography (TCD) was performed using 2 and $4 \mathrm{MHz}$ frequencies transducers. The flow velocities of the internal carotid artery (ICA), middle cerebral artery (MCA), posterior cerebral artery (PCA), ophthalmic artery (OA) and NO plasma concentration were assessed 2 hours after migraine attacks. The patients with cardiovascular risk factors were excluded. Exclusion criteria were age above 40 , hypertension, diabetes mellitus, obesity, dyslipidemia, chronis alcoholism, current smoking, and any previous cardio-vascular or cerebro-vascular events. Total nitrate and nitrite (NOx) were measured in plasma by Griess reaction using commercial kits (17).

\section{Statistical analysis}

The comparison between groups was made with Student test for normal distributed data, with ManWhitney test for non-normal distributed data, and Chi-square test for non-parametric data. Spearman test were applied for correlation calculation for non-normal distributed data, and Pearson test for correlation of normal distributed data. p $<0.05$ were considered significant. The correlations were made between flow velocities values in cerebral vessels assessed by TDU and NOx level.

\section{RESULTS}

The NO synthesis was significantly increased compared to the control group (Table1). The flow velocities significantly decreased in internal carotid artery, middle cerebral artery, and posterior cerebral artery, and ophthalmic artery flow velocities were found to be increased (Table2). The correlations between pathological flow velocities and NOx plasma concentration were significant for MCAPSFV and OA-PSFV (Table 3).

Significant monotonic positive relationship was identified for NOx with MCA-PSFV and, a negative monotonic relationship between NOx and OAPSFV. No significant relationship was identified between NOx and other arteries PSFV in study group patients.

\section{DISCUSSIONS}

Our study group presented a significant increased NOx synthesis that proved that endothelial 
TABLE 1. Baseline characteristics of patients with OM compared to control. The values are expressed as Mean (SD) - for normal distributed data, median (quartiles) for non-normal distributed data, and number of subjects for non-parametric data.

\begin{tabular}{|l|c|c|c|}
\hline Parameter & $\begin{array}{c}\text { Group 1 (Control) } \\
\mathbf{N = 3 5}\end{array}$ & $\begin{array}{c}\text { Group 2 (Study) } \\
\mathbf{N}=\mathbf{3 7}\end{array}$ & P value \\
\hline Age (years) & $38.5(36.5-41)$ & $37.25(35-40)$ & NS \\
\hline BMI (Quetelet formula)* & $23.72(23-25)$ & $25.3(25.5-27)$ & $p<0.01$ \\
\hline TAS (mmHg)* & $115(113-124)$ & $120(118-122)$ & NS \\
\hline TAD (mmHg)** & $75.5(74-78)$ & $80.23(5.45)$ & NS \\
\hline Glycemia (mg\%)* & $90(88-97)$ & $86(80-98)$ & NS \\
\hline Smoking status - former smoking /non-smoking*** & $10 / 25$ & $18 / 19$ & $p<0.05$ \\
\hline Alcohol consumption (Yes/No) & $5 / 50$ & $11 / 62$ & NS \\
\hline CRP (mg\%)* & $0.45(0.15)$ & $1.12(0.19)$ & $p<0.0001$ \\
\hline Fibrinogen (mg\%) & $200.45(150-235)$ & $260.75(225-280)$ & NS \\
\hline NOx ( $\mu$ mol/L) & $35.25(40.37-50.23)$ & $55.23(51.75-57.23)$ & $p<0.001$ \\
\hline LDL -CST (mg\%)* & $111(105-130)$ & $121(115-130)$ & NS \\
\hline HDL-CST (mg\%)* & $41(35.5-45)$ & $38(35-42)$ & NS \\
\hline TG (mg\%)* & $135(125-145)$ & $140(130-150)$ & NS \\
\hline
\end{tabular}

*Man -Whitney test, ${ }^{* *}$ t-test, ${ }^{* *}$ Chi-square test, NS - non-significant

TABLE 2. Peak systolic flow velocities (PSFV) in internal carotid artery (ICA), middle cerebral artery (MCA), posterior cerebral artery (PCA), and ophthalmic artery (OA) - migraine patients (side with visual symptoms) compared to control group.

\begin{tabular}{|c|c|c|c|}
\hline Parameter & $\begin{array}{c}\text { Group 1 (Control) } \\
\mathbf{N}=\mathbf{3 5}\end{array}$ & $\begin{array}{c}\text { Group 2 (Study) } \\
\mathbf{N}=\mathbf{3 7}\end{array}$ & P value \\
\hline ICA- PSFV* & $65.25(60.75-67.25)$ & $54.35(52.17-57.27)$ & $\mathrm{P}<0.05$ \\
\hline MCA-PSFV* & $64.24(62.25-66-85)$ & $50.25(48.45-52.75)$ & $\mathrm{P}<0.01$ \\
\hline PCA-PSFV* & $42.45(40.24-44.45)$ & $40.75(38.23-42.75)$ & $\mathrm{NS}$ \\
\hline OA-PSFV* & $43.45(41.23-45.40)$ & $48.44(45.66-52.45)$ & $\mathrm{P}<0.01$ \\
\hline
\end{tabular}

*Man -Whitney test

Relationship between NOx, and flow velocities (FV) in internal carotid artery (ICA), middle cerebral artery (MCA), posterior cerebral artery (PCA), and ophthalmic artery (OA)

TABLE 3. Significant monotonic relationship expressed as Spearman's rank correlation ( $p$ values) in study group.

\begin{tabular}{|l|c|c|c|c|}
\hline & $\begin{array}{c}\text { NOx and } \\
\text { ICA-PSFV }\end{array}$ & $\begin{array}{c}\text { NOx and } \\
\text { MCA-PSFV }\end{array}$ & $\begin{array}{c}\text { NOx and } \\
\text { PCA-PSFV }\end{array}$ & $\begin{array}{c}\text { NOx and } \\
\text { OA-PSFV }\end{array}$ \\
\hline$p$ value & NS & $p<0.05$ & NS & $p<0.05$ \\
\hline
\end{tabular}

function is normal. Our previous study also demonstrated significant changes of NOx plasma level associated with ophthalmic migraine (18). Due to aging process the endothelial dysfunction is leading to decrease of NOx synthesis $(19,20)$ and a low bioavailability of nitric oxide to maintain the physiological cerebral vasodilatation (21). Increased NOx synthesis produce vasodilatation in large arteries demonstrated by systolic flow velocities in our study group. Decreasing flow velocities in ophthalmic artery showed a hemodynamic perturbation with possible vasospasm in ophthalmic artery. There are other factors that may contribute to he- modynamic perturbation in ophthalmic artery as are the presence of inflammatory processes (demonstrated by significant CRP increasing in study group). Our results demonstrated that the decreasing ophthalmic artery diameter due to vasospasm may lead to visual symptoms associated migraine. The correlation on NOx synthesis with increased diameter of large arteries demonstrated that the vasodilatation of these vessels can lead to decrease of blood flow in ophthalmic artery possible due to "vascular steal syndrome". A genetic predisposition to this vasomotor response, to increased glutamate neurotransmission and proper synaptic plasticity may play an important role (22).

Our results that demonstrated the increases proinflammatory plasma markers as is $\mathrm{C}$ reactive protein, brings the connection between the existence of inflammatory status in ophthalmic migraine patients and increased synthesis of nitric oxide, due to inflammation. The presence of an inflammatory 
mechanism may lead to central sensitization and under repetition even that is low-intensity stimulation induces a cephalic allodynia (23). Clinically, central sensitization is manifested as decreased pain threshold and exaggerated pain response that is referred outside the original pain site (24). The application of inflammatory substances on the exposed rat dura, which activates the trigeminovascular pathway induces long-lasting sensitization in dorsal horn neurons that receive intracranial input from the dura and extracranial input from the periorbital area (25). Increased nitric oxide level may contribute to this mechanism. The associated vascular and neural contribution may explain some pathophysiological aspects in migraine mechanism (26). The efficiency of non-steroidal anti-inflammatory drug in treatment of migraine attack may demonstrate de correlation between nitric oxide synthesis augmentation (due to activation on iNOS) and increasing the prostaglandin synthesis $(27,28)$. Administration of parenteral COX1/COX2 inhibitors may block sensitization in meningeal nociceptors and suppressed ongoing sensitization in spinal trigeminovascular neurons (29). Furthermore, the vasodilatation induced by increased NO synthesis, may lead to increased blood-brain barrier permeability and inflammation associated hyperalgesia (30). These two opposite diameters changes may underlie to pain associated with vasodilatation and visual symptoms associated with ophthalmic artery constriction. That evidence may lead to possibility for new strategies of migraine treatment directed to the inhibition or decreasing of NO synthesis.

\section{CONCLUSIONS}

Hemodynamic perturbation in ophthalmic artery is an important pathophysiologic mechanism associated with ophthalmic migraine. The patients' age in our study group can contribute to higher synthesis of NO. The flow velocity status may reflect the vasomotor response and endothelial reactivity induced by NO synthesis. Further studies on the vasomotor response are necessary for evaluation of this pathophysiological mechanism implication in ophthalmic migraine.

\section{REFERENCES}

1. Quillon A., Fromy B., Debret R. Endothelium microenvironment sensing leading to nitric oxide mediated vasodilation: a review of nervous and biomechanical signals. Nitric Oxide. 2015 Feb 15; 45:20-6. Epub 2015 Jan 28.

2. Weidinger A., Kozlov A.V. Biological Activities of Reactive Oxygen and Nitrogen Species: Oxidative Stress versus Signal Transduction. Biomolecules. 2015 Apr 15; 5(2):472-484.

3. Bassenge $E$. Clinical relevance of endothelium-derived relaxing factor (EDRF). Br J Clin Pharmacol. 1992; 34 Suppl 1:37S-42S.

4. Sessa W.C. The nitric oxide synthase family of proteins. J Vasc Res. 1994 May-Jun; 31(3):131-43.

5. Salvemini D., Kim S.F., Mollace V. Reciprocal regulation of the nitric oxide and cyclooxygenase pathway in pathophysiology: relevance and clinical implications. Am J Physiol Regul Integr Comp Physiol. 2013 Apr 1; 304(7):R473-87.Epub 2013 Feb 6.

6. Salvemini D., Little J.W., Doyle T., Neumann W.L. Roles of reactive oxygen and nitrogen species in pain. Free Radic Biol Med. 2011 Sep 1;51(5):951-66. doi: 10.1016/j.freeradbiomed.2011.01.026. Epub 2011 Jan 28.

7. Messlinger K., Lennerz J.K., Eberhardt M., Fischer M.J. CGRP and $\mathrm{NO}$ in the trigeminal system: mechanisms and role in headache generation. Headache. 2012 Oct; 52(9):1411-27.Epub 2012 Jul 12.

8. Bulboaca C.A., Bulboaca A., Prodan C. Platelet activation and nitric oxide synthesis in patients with transient ischemic attack, Neurology, 2011, 76(9), Suppl 4:A424-425.

9. Lerman A., Burnett J.C. Jr. Intact and altered endothelium in regulation of vasomotion. Circulation. 1992 Dec; 86(6 Suppl):III12-19.

10. Pacher P., Beckman J.S., Liaudet L. Nitric oxide and peroxynitrite in health and disease. Physiol Rev. 2007 Jan; 87(1):315-424.

11. Hilz M.J., Stemper B., Heckmann J.G., Neundörfer B. Mechanisms of cerebral autoregulation, assessment and interpretation by means of transcranial doppler sonography. Fortschr Neurol Psychiatr. 2000 Sep; 68(9):398-412.

12. Naqvi J., Yap K.H., Ahmad G., Ghosh J. Transcranial Doppler ultrasound: a review of the physical principles and major applications

in critical care. Int J Vasc Med. 2013; 2013:629378.

Epub 2013 Dec 12.

13. Guidetti D., Rota E., Morelli N., Immovilli P. Migraine and stroke: „vascular" comorbidity. Front Neurol. 2014 Oct 8; 5:193.

14. Schillaci G., Sarchielli P., Corbelli I., Pucci G., Settimi L., Mannarino M.R., Calabresi P, Mannarino E. Aortic stiffness and pulse wave reflection in young subjects with migraine: A case-control study. Neurology. 2010 Sep 14; 75(11):960-6.

15. Purdy R.A. The role of the visual system in migraine: an update. Neurol Sci. 2011 May; 32 Suppl 1:S89-93.

16. Schankin C.J., Maniyar F.H., Sprenger T., Chou D.E., Eller M., Goadsby P.J. The relation between migraine, typical migraine aura and „visual snow". Headache. 2014 Jun; 54(6):957-66. Epub 2014 May 9.

17. Tsikas D. Analysis of nitrite and nitrate in biological fluids by assays based on the Griess reaction: appraisal of the Griess reaction in the L-arginine/nitric oxide area of research. J Chromatogr B Analyt Technol Biomed Life Sci. 2007 May 15;851(1-2):51-70. Epub 2006 Sep 6.

18. Bulboaca A.E. The correlation of nitric oxide synthesis and visual evoked potential in ophthalmic migraine patients, Journal of Neurology 2014, 261 Suppl 1: S407-S407

19. Gradinaru D., Borsa C., Ionescu C., Prada G.I. Oxidized LDL and NO synthesis-Biomarkers of endothelial dysfunction and ageing. Mech Ageing Dev. 2015 Mar 21.

20. Donato A.J., Morgan R.G., Walker A.E., Lesniewski L.A. Cellular and molecular biology of aging endothelial cells. J Mol Cell Cardiol. 2015 Feb 2.

21. Pretnar-Oblak J. Cerebral endothelial function determined by cerebrovascular reactivity to L-arginine. Biomed Res Int. 2014; 2014:601515. Epub 2014 Apr 17.

22. Burstein R., Noseda R., Borsook D. Migraine: multiple processes, complex pathophysiology. J Neurosci. 2015 Apr 29; 35(17):6619-29.

23. Boyer N., Dallel R., Artola A., Monconduit L. General trigeminospinal central sensitization and impaired descending pain 
inhibitory controls contribute tomigraine progression. Pain. $2014 \mathrm{Jul}$; 155(7):1196-205. Epub 2014 Mar 12.

24. Burstein R., Jakubowski M., Rauch S.D. The science of migraine. J Vestib Res. 2011; 21(6):305-14.

25. Burstein R., Yamamura H., Malick A., Strassman A.M. Chemical stimulation of the intracranial dura induces enhanced responses to facial stimulation in brain stem trigeminal neurons. Journal of Neurophysiology. 1998; 79:964-982.

26. Silberstein S.D. Migraine pathophysiology and its clinical implications. Cephalalgia. 2004; 24 Suppl 2:2-7.

27. Manzoni G.C., Torelli P. Symptomatic treatment of migraine: from scientific evidence to patient management, Neurol Sci, 2014,35 (Suppl 1):11-15
28. Belvis R., Mas N., Aceituno A. Migraine attack treatment : a tailor-made suit, not one size fits all. Recent Pat CNS Drug Discov. 2014 Apr; 9(1):26-40.

29. Jakubowski M., Levy D., Goor-Aryeh I., Collins B., Bajwa Z., Burstein R. Terminating migraine with allodynia and ongoing central sensitization using parenteral administration of COX1/COX2 inhibitors. Headache. 2005 Jul-Aug; 45(7):850-61.

30. DosSantos M.F., Holanda-Afonso R.C., Lima R.L., DaSilva A.F., Moura-Neto V. The role of the blood-brain barrier in the development and treatment of migraine and other pain disorders. Front Cell Neurosci. 2014 Oct 8; 8:302. 